\title{
Tendências em dez anos das condições de saúde de idosos brasileiros: evidências da Pesquisa Nacional por Amostra de Domicílios (1998, 2003, 2008)
}

\author{
10-year trends in the health of Brazilian elderly: evidence from \\ the National Household Sample Survey (PNAD 1998, 2003, 2008)
}

\author{
Maria Fernanda Lima-Costa ${ }^{1}$ \\ Divane Leite Matos ${ }^{1}$ \\ Vitor Passos Camargos ${ }^{2}$ \\ James Macinko ${ }^{3}$
}

${ }^{1}$ Núcleo de Estudos em Saúde Pública e

Envelhecimento da Fundação Oswaldo Cruz,

Centro de Pesquisas René Rachou, Belo Horizonte,

Brasil e Universidade Federal de Minas Gerais. Av. Augusto de Lima 1715. 30190-002 Belo Horizonte MG. lima-costa@ cpqrr.fiocruz.br

${ }^{2}$ Programa de Pó Graduação em Saúde Pública da Universidade Federal de Minas Gerais, Faculdade de Medicina. ${ }^{3}$ Department of Nutrition, Food Studies \& Public Health, New York University, New York, USA.
Abstract Data from a representative national household survey were used to examine 10-year trends (1998-2008) in health conditions of Brazilian elderly. A random sample of 105,254 individuals aged $\geq 60$ years participated. The mean age was 69 years and 56\% were women. There was a gradual increase in the prevalence of good or excellent self-rated health (39.3\%, 43.5\% and 45.0\%, in 1998, 2003 and 2008, respectively) and a decrease in self-reported arthritis, heart disease, and depression. The prevalence of self-reported hypertension $(43.9 \%, 48.8 \%$, and $53.3 \%$, respectively) and diabetes $(10.3 \%, 13.0 \%$ and $16.1 \%$ respectively) increased sharply. The prevalence of inability to perform activities of daily living (eating, bathing or using the toilet) remained stable $(6.5 \%, 6.4 \%$ and $6.9 \%$, respectively). The achievement of three or more physician visits over the previous 12 months increased by $21 \%$ from 1998 to 2008. Hospitalization decreased by $10 \%$ in the corresponding period. The above mentioned trends persisted after adjustments for age and sex. The results showed improvements in some health dimensions of the elderly, but not all. Changes in the use of health services occurred as expected with the expansion of primary healthcare activities in Brazil.

Key words Aging, Elderly, Health survey, Multidimensional, Trends
Resumo Foram examinadas as tendências em saúde da população idosa brasileira utilizando-se dados da Pesquisa Nacional por Amostra de Domicílios (1998-2008). O estudo incluiu uma amostra probabilística de 105.254 pessoas com $\geq 60$ anos de idade. A média da idade foi de 69 anos e $56 \%$ eram mulheres. Houve um aumento gradativo da boa autoavaliação da saúde (39,3\%, 43,5\% e 45,0\% em 1998, 2003 e 2008, respectivamente) e uma diminuição na prevalência de artrite, doença do coração e depressão autorreferidas. A prevalência da hipertensão (43.9\%, 48.8\% e 53.3\%, respectivamente) e do diabetes autorreferidos (10.3\%, 13.0\% e $16.1 \%$ respectivamente) aumentou acentuadamente. A prevalência da incapacidade para realizar atividades da vida diária (alimentar-se, tomar banho ou ir ao banheiro) permaneceu estável $(6.5 \%, 6.4 \%$ e $6.9 \%$, respectivamente). A realização de três ou mais consultas médicas nos 12 meses precedentes aumentou 21\% entre 1998 e 2008. As hospitalizações diminuíram $10 \%$ no mesmo período. Essas tendências foram independentes do sexo e da idade. Os resultados mostram melhora em algumas dimensões da saúde dos idosos, mas não em todas. As mudanças no uso de serviços de saúde ocorreram como esperado em decorrência da expansão das atividades de atenção primária no Brasil.

Palavras-chave Envelhecimento, Idoso, Inquérito de saúde, Multi-dimensional, Tendências 


\section{Introdução}

O envelhecimento da população é um fenômeno global, que vem ocorrendo em todas as regiões e países do mundo, com exceção daqueles em extrema pobreza. Essa mudança demográfica é conseqüência de uma impressionante redução da fertilidade e da mortalidade observada ao longo do século $\mathrm{XX}^{1}$. O Brasil está envelhecendo rapidamente. Na última década, a população brasileira com idade igual ou superior a 60 anos cresceu 2,5 vezes mais $(36 \%)$ do que a mais jovem $(14 \%)^{2}$. Entretanto, o conhecimento das tendências no longo prazo das condições de saúde dos idosos no país ainda é restrito a informações sobre a mortalidade ${ }^{3}$.

As tendências das condições de saúde da população idosa vêm recebendo atenção crescente. Estudos sobre o tema têm sido conduzidos tanto em países mais ricos, nos quais o envelhecimento populacional foi mais precoce ${ }^{4-7}$, quanto em países de média renda, onde essa mudança demográfica é mais recente ${ }^{8-10}$. Até cerca de 20 anos atrás, acreditava-se que as tendências da mortalidade representassem adequadamente as condições de saúde da população idosa. Como a mortalidade estava diminuindo, assumia-se que as condições de saúde estavam melhorando ${ }^{4}$. Entretanto, logo ficou claro que a redução da mortalidade não implicava em anos adicionais com saúde e autonomia. Além disso, constatou-se que a saúde da população idosa é multidimensional e que a melhora em uma dimensão não se reflete necessariamente em outra ${ }^{4}$. Assim, formou-se um consenso de que outros indicadores são importantes para se avaliar as tendências em saúde dessa população. Entre esses indicadores, os mais comumente utilizados são a autoavaliação da saúde, a prevalência de doenças e de condições crônicas e a capacidade funcional ${ }^{4-9}$.

Os indicadores acima mencionados são baseados em informações obtidas em inquéritos populacionais, o que torna limitado o seu uso aos países que produzem essas informações com alguma periodicidade. $\mathrm{O}$ Brasil possui inquérito de saúde, conduzido como parte da Pesquisa Nacional por Amostras de Domicílios (PNAD) em 1998, 2003 e 2008, com amostra suficiente para permitir estimativas com boa precisão para a população idosa. Resultados do inquérito de 1998 mostraram que as prevalências de algumas doenças crônicas autorreferidas e da incapacidade funcional entre idosos eram razoavelmente semelhantes às de populações idosas em países de alta renda, e que o uso de serviços de saúde estava dentro das variações observadas internacionalmente $^{11}$. Posteriormente, foram comparados os resultados dos inquéritos de 1998 e 2003. Essa comparação mostrou melhora na autoavaliação da saúde e na capacidade funcional, redução na prevalência de artrite ou reumatismo, e aumento do número de consultas médicas. Por outro lado, observou-se um aumento na prevalência da hipertensão arterial e do diabetes, autoreferido ${ }^{10}$. Essa avaliação, entretanto, não foi suficiente para estabelecer claramente uma tendência, uma vez que a pesquisa baseou-se em duas medidas, obtidas com intervalo de cinco anos.

A disponibilização recente dos dados da PNAD 2008 permite, pela primeira vez, investigar as tendências no longo prazo das condições de saúde da população idosa brasileira, com outras informações que não a mortalidade. $\mathrm{O}$ objetivo do presente trabalho é investigar essas tendências, considerando-se a autoavaliação da saúde, a prevalência de doenças e de condições crônicas auto referidas, a capacidade funcional e o uso de serviços de saúde.

\section{Metodologia}

\section{População estudada}

A PNAD é um inquérito com abrangência nacional, realizado anualmente pela Fundação Instituto Brasileiro de Geografia e Estatística (IBGE), com o objetivo de obter informações sobre características demográficas, habitação, educação, trabalho e rendimentos da população brasileira. Em 1998, 2003 e 2008, a PNAD incluiu um suplemento com perguntas sobre as condições de saúde e os aspectos a ela relacionados. A PNAD é baseada numa amostra probabilística de domicílios obtida em três estágios de seleção. No primeiro estágio, é feita a seleção dos municípios, que são classificados em autorrepresentativos, com probabilidade 1 de pertencer à amostra, e em não autorrepresentativos, com probabilidade de pertencer à amostra proporcional à população residente. No segundo, são selecionados os setores censitários, cuja probabilidade de inclusão é proporcional ao número de domicílios existentes no setor. No terceiro, os domicílios são amostrados em cada setor censitário e seus moradores são elegíveis para a entrevista face a face. Em 1998, 2003 e 2008, 344.975, 384.834 e 391.868 pessoas participaram da PNAD, respectivamente. Para o presente trabalho, foram selecionados todos os participantes da PNAD, nos 
anos acima mencionados, com idade igual ou superior a 60 anos. Maiores detalhes podem ser vistos em outras publicações ${ }^{12-14}$.

\section{Variáveis}

As variáveis dependentes deste estudo incluíram quatro dimensões ${ }^{4}$ : (1) autoavaliação da saúde; (2) capacidade funcional; (3) doenças e condições crônicas auto referidas e (4) indicadores de uso de serviços de saúde (consultas médicas, hospitalizações e uso exclusivo do Sistema Único de Saúde [SUS]). A variável independente do estudo foi o ano no qual o inquérito foi realizado.

A informação sobre autoavaliação da saúde foi obtida por meio da pergunta "De um modo geral, considera seu próprio estado de saúde como...", com cinco possibilidades de respostas, variando entre excelente e muito ruim. A incapacidade funcional foi definida pelo relato de muita dificuldade ou total incapacidade para realizar três atividades da vida diária (AVD): alimentar-se, tomar banho ou usar o toalete. A condição de portador de doenças crônicas foi definida pela resposta à pergunta "Você tem (a doença)" no inquérito de 1998 e pela resposta à pergunta "Algum médico ou profissional de saúde já disse que você tem (a doença?)" nos inquéritos subsequentes. Os indicadores do uso de serviços de saúde incluíram o número de consultas médicas realizadas nos últimos 12 meses e a ocorrência de uma ou mais internações hospitalares ocorridas no mesmo período. O uso exclusivo do SUS foi definido pelo relato de não possuir cobertura pela medicina suplementar; aqueles que relataram ter plano privado de saúde somente para assistência odontológica foram considerados como usuários exclusivos do SUS.

\section{Análise dos dados}

As estimativas das prevalências foram feitas, incorporando-se os pesos e o efeito do delineamento da amostra ${ }^{15}$. As análises das associações entre o ano e os indicadores das condições de saúde e do uso de serviços de saúde foram baseadas em Razões de Prevalência (RP) e intervalos de confiança de 95\% (IC 95\%) ajustadas por idade (variável contínua) e sexo, que foram estimadas por meio da regressão de Poisson. Todas as análises foram realizadas utilizando-se os procedimentos para amostras complexas do pacote estatístico Stata (College Station, Texas, USA), versão 11.0 .

\section{Resultados}

A presente análise incluiu 105.254 idosos, assim distribuídos: 28.943 participantes do inquérito de 1998, 35.042 do de 2003 e 41.269 do de 2008. A proporção de mulheres não diferiu nos três inquéritos ( $\mathrm{p}>0,05): 55,5 \%$ (IC 95\% 55,0-56,0), $55,9 \%$ (IC95\% 55,5-56,4) e 56,2\% (IC95\% 55,8$56,6)$, respectivamente. A média da idade foi mais baixa no ano inicial (69,5 anos; IC95\% 69,7-69,6) e mais alta nos subsequentes $(69,8$; IC 95\% 69,569,9 em 2003) e 69,9 (IC95\% 69,8-70,0 em 2008) ( $\mathrm{p}<0,05$ para comparações com 1998).

$\mathrm{Na}$ Tabela 1 estão apresentados os resultados da análise das tendências dos indicadores das condições de saúde e do uso de serviços de saúde nos anos de 1998, 2003 e 2008. A prevalência da autoavaliação da saúde como boa ou excelente aumentou entre 1998 e 2003 e entre 1998 e 2008, assim como a prevalência de hipertensão, do diabetes e da realização de três ou mais consultas médicas nos 12 meses precedentes, independentemente da idade e do sexo. Por outro lado, nos períodos correspondentes houve diminuição da prevalência de artrite ou reumatismo, de doenças do coração, de depressão, de hospitalizações nos últimos 12 meses e do uso exclusivo do SUS. A prevalência de incapacidade funcional não mudou significativamente no período estudado.

Na Figura 1 estão apresentadas as tendências da autoavaliação da saúde como boa ou excelente, segundo a faixa etária. Observa-se um aumento gradual e consistente da boa avaliação da saúde nas faixas etárias de 60-69, 70-79 e 80+ anos de idade $(\mathrm{p}<0.001$ em cada grupo). Não houve mudanças na distribuição por idade da incapacidade funcional ao longo do período estudado. Essas prevalências permaneceram estáveis tanto na faixa etária de 60-69 anos (3,5 [IC95\% 3,1-3,8] em 1998, 3,1 [2,8-3,4] em 2003 e $3,5$ [3,2-3,8] em 2008; $p=0.203)$, quanto nas faixas etárias de 70-79 anos (7,4 [6,8-8,1] em 1998, $7,0[6,4-7,6]$ em 2003 e 7,2 [6,6-7,7] em 2008; $\mathrm{p}=0.629)$ e $80+\operatorname{anos}(18,8 \%$ [IC 95\% 17,1-20,4] em 1998, 19,5\% [18,0-21,9] em 2003 e 20,2 [18,821,5] em 2008; p=0,421). Como esperado, em cada um dos anos estudados, a prevalência da incapacidade funcional foi mais alta nas faixas etárias superiores, sobretudo na mais velha.

Como pode ser observado na Figura 2, houve aumento da realização de três ou mais consultas médicas entre 1998 e 2003 nas três faixas etárias idosas ( $\mathrm{p}<0.001$ em cada grupo). Em cada um dos anos estudados, observou-se aumento progressivo do número de consultas médicas com a idade. 
Na Figura 3 estão apresentadas as tendências das hospitalizações. A proporção de hospitalizações diminuiu entre 1998 e 2008 na faixa etária de 60-69 anos $(\mathrm{p}<0,001)$, mas não nas faixas etárias de 70-79 $(\mathrm{p}=0.089)$ e $80+\operatorname{anos}(\mathrm{p}=0.802)$. De maneira geral, a proporção de hospitalizações foi mais alta nas faixas etárias superiores.
Como pode ser visto na Tabela 2, a melhora da autoavaliação da saúde ocorreu de forma consistente em todas as regiões brasileiras, tanto entre 2003 e 1998, quanto entre 2008 e 1998. Nos períodos correspondentes, não foram observadas mudanças na prevalência da incapacidade funcional nas cinco regiões. A realização de três

Tabela 1. Análise das tendências dos indicadores das condições de saúde e do uso de serviços de saúde entre idosos brasileiros (PNAD, 1998, 2003, 2008)

\begin{tabular}{|c|c|c|c|c|c|}
\hline \multirow{2}{*}{ Indicadores } & \multirow[b]{2}{*}{1998} & \multirow[b]{2}{*}{2003} & \multirow[b]{2}{*}{2008} & \multicolumn{2}{|c|}{ RP (IC 95\%) } \\
\hline & & & & 2003 vs. 1998 & 2008 vs. 1998 \\
\hline Autoavaliação da saúde boa ou muito boa & 39,3 & 43,5 & 45,0 & $1,11(1,08-1,15)$ & $1,15(1,12-1,19)$ \\
\hline $\begin{array}{l}\text { Incapacidade para realizar atividades da vida } \\
\text { diária }{ }^{1}\end{array}$ & 6,5 & 6,4 & 6,9 & $0,95(0,88-1,03)$ & $1,00(0,92-1,07)$ \\
\hline Hipertensão arterial & 43,9 & 48,8 & 53,3 & $1,11(1,08-1,13)$ & $1,21(1,18-1,23)$ \\
\hline Diabetes & 10,3 & 13,0 & 16,1 & $1,26(1,19-1,33)$ & $1,56(1,48-1,64)$ \\
\hline Artrite ou reumatismo & 37,5 & 27,3 & 24,2 & $0,72(0,70-0,75)$ & $0,64(0,62-0,66)$ \\
\hline Doença do coração & 19,0 & 17,4 & 17,3 & $0,91(0,87-0,95)$ & $0,90(0,86-0,94)$ \\
\hline Depressão & 12,1 & 9,7 & 9,2 & $0,80(0,75-0,86)$ & $0,76(0,71-0,81)$ \\
\hline $\begin{array}{l}\text { Número de consultas médicas nos últimos } \\
12 \text { meses (três ou mais) }\end{array}$ & 44,3 & 51,0 & 54,0 & $1,15(1,12-1,18)$ & $1,21(1,19-1,24)$ \\
\hline $\begin{array}{l}\text { Hospitalizações nos últimos } 12 \text { meses } \\
\text { (uma ou mais) }\end{array}$ & 13,6 & 12,7 & 12,3 & $0,93(0,88-0,98)$ & $0,89(0,85-0,94)$ \\
\hline $\begin{array}{l}\text { Usuários exclusivos do Sistema Único } \\
\text { de Saúde (SUS) }\end{array}$ & 74,7 & 72,2 & 71,9 & $0,97(0,95-0,98)$ & $0,96(0,95-0,98)$ \\
\hline
\end{tabular}

RP (IC95\%): Razão de prevalência e intervalo de confiança de 95\% ajustada por sexo e idade, estimada por meio da regressão de Poisson por sexo e idade. ${ }^{1}$ Alimentar-se, tomar banho ou ir ao banheiro

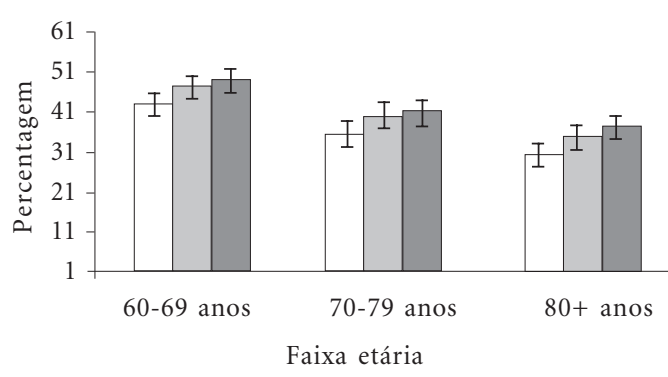

$\square 1998 \square 2003 \square 2008$

Figura 1. Distribuição da autoavaliação da saúde como boa ou excelente entre idosos brasileiros, segundo a faixa etária e o ano (PNAD, 1998, 2003, 2008)

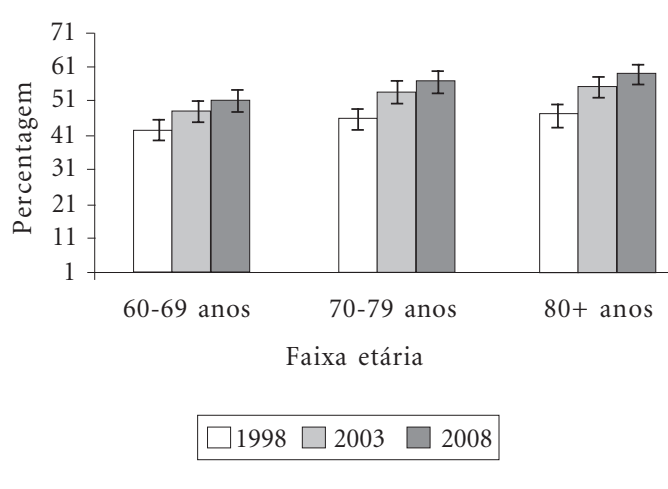

Figura 2. Distribuição de consultas médicas (três ou mais) entre idosos brasileiros, segundo a faixa etária e o ano (PNAD, 1998, 2003, 2008) 
ou mais consultas médicas nos últimos 12 meses aumentou significativamente em todas as regiões do país, tanto nas comparações entre 2003 e 1998, quanto entre 2008 e 1998.

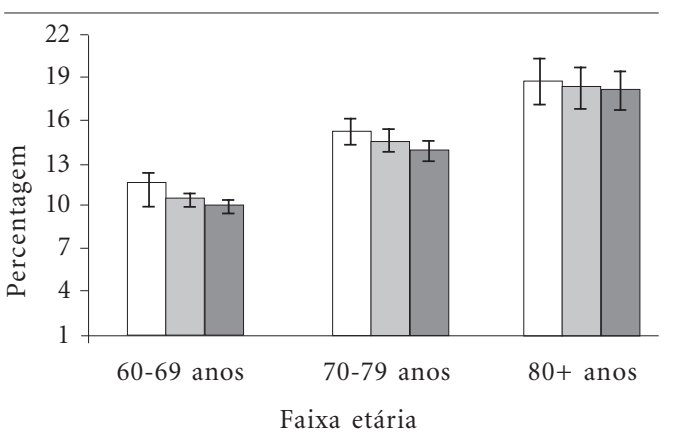

$\square 1998 \square 2003 \square 2008$

Figura 3. Distribuição das hospitalizações (uma ou mais) entre idosos brasileiros, segundo a faixa etária e o ano (PNAD, 1998, 2003, 2008)

\section{Discussão}

Os resultados deste trabalho mostram tendências diferentes entre as várias dimensões da saúde dos idosos. Durante a década estudada, foi observada uma melhora significativa na autoavaliação da saúde e uma diminuição na prevalência de algumas doenças ou condições crônicas. Mas a prevalência da incapacidade funcional permaneceu estável e observou-se aumento na da hipertensão arterial e na do diabetes.

A autoavaliação da saúde é uma medida global que capta o bem estar físico, mental e social do indivíduo ${ }^{16,17}$. Ela tem sido amplamente recomendada como um dos indicadores mais importantes para o monitoramento das condições de saúde da população ${ }^{18,19}$. Resultados recentes de inquéritos populacionais em países de média renda mostraram que a autoavaliação da saúde melhorou entre idosos chineses ${ }^{8}$, mas piorou entre idosos sulcoreanos ${ }^{9}$. Os resultados do presente trabalho mostram uma melhora consistente na autoavaliação de saúde dos brasileiros. Essa melhora foi observada em todas as regiões do país, assim como nas três faixas etárias idosas.

Tabela 2. Autoavaliação da saúde, incapacidade funcional e número de consultas médicas entre idosos brasileiros por macro-região, segundo o ano (PNAD, 1998, 2003, 2008)

\begin{tabular}{|c|c|c|c|c|c|}
\hline \multirow{2}{*}{ Indicadores por macro-região } & \multirow[b]{2}{*}{1998} & \multirow[b]{2}{*}{2003} & \multirow[b]{2}{*}{2008} & \multicolumn{2}{|c|}{ RP (IC 95\%) } \\
\hline & & & & 2003 vs. 1998 & 2008 vs. 1998 \\
\hline \multicolumn{6}{|l|}{ Autoavaliação da saúde boa ou muito boa } \\
\hline Norte & 28,9 & 36,9 & 37,3 & $1,28(1,12-1,46)$ & $1,29(1,14-1,45)$ \\
\hline Nordeste & 31,7 & 34,9 & 37,1 & $1,02(1,02-1,19)$ & $1,17(1,10-1,25)$ \\
\hline Sudeste & 44,8 & 48,7 & 50,1 & $1,09(1,05-1,14)$ & $1,13(1,09-1,17)$ \\
\hline Sul & 39,9 & 45,4 & 46,3 & $1,14(1,06-1,23)$ & $1,22(1,11-1,34)$ \\
\hline Centro-Oeste & 33,9 & 41,3 & 42,2 & $1,37(1,18-1,59)$ & $1,26(1,15-1,37)$ \\
\hline \multicolumn{6}{|c|}{$\begin{array}{l}\text { Incapacidade para realizar atividades da vida } \\
\text { diária }{ }^{1}\end{array}$} \\
\hline Norte & 6,9 & 6,2 & 6,0 & $0,88(0,64-1,21)$ & $0,84(0,64-1,11)$ \\
\hline Nordeste & 7,7 & 7,8 & 7,9 & $0,98(0,86-1,13)$ & $0,99(0,88-1,13)$ \\
\hline Sudeste & 5,9 & 5,6 & 6,4 & $0,93(0,82-1,06)$ & $1,00(0,89-1,13)$ \\
\hline Sul & 6,2 & 6,4 & 7,2 & $1,00(0,85-1,18)$ & $1,10(0,85-1,18)$ \\
\hline Centro-Oeste & 7,0 & 6,2 & 6,6 & $0,87(0,66-1,13)$ & $0,86(0,66-1,12)$ \\
\hline \multicolumn{6}{|c|}{$\begin{array}{l}\text { Número de consultas médicas nos últimos } \\
12 \text { meses (três ou mais) }\end{array}$} \\
\hline Norte & 35,5 & 45,3 & 47,8 & $1,27(1,14-1,42)$ & $1,35(1,21-1,50)$ \\
\hline Nordeste & 35.0 & 43,6 & 48,0 & $1,24(1,18-1,31)$ & $1,37(1,30-1,44)$ \\
\hline Sudeste & 50,5 & 56,3 & 59,0 & $1,11(1,07-1,14)$ & $1,16(1,12-1,20)$ \\
\hline Sul & 44,2 & 50,2 & 51,5 & $1,13(1,07-1,24)$ & $1,16(1,10-1,22)$ \\
\hline Centro-Oeste & 42,1 & 48,5 & 52,8 & $1,15(1,12-1,49)$ & $1,24(1,16-1,33)$ \\
\hline
\end{tabular}

RP (IC95\%): Razão de prevalência e intervalo de confiança de 95\% ajustada por sexo e idade, estimada por meio da regressão de Poisson por sexo e idade. ${ }^{1}$ Alimentar-se, tomar banho ou ir ao banheiro. 
A capacidade funcional é provavelmente a dimensão mais estudada da saúde dos idosos. Isso ocorre porque, além do sofrimento pessoal e familiar, ela aumenta a demanda por serviços médicos e sociais, com custos correspondentes. A capacidade funcional é geralmente aferida em inquéritos populacionais pelo grau de dificuldade (ou pela necessidade de ajuda) para a realização de atividades da vida diária (AVD) e/ou atividades instrumentais da vida diária (AIVD). As primeiras incluem a habilidade para alimentarse, vestir-se, tomar banho, usar o toalete, levantar-se (ou transferir-se da cama para uma cadeira) e às vezes caminhar em um cômodo no mesmo andar. As AIVD incluem atividades como administrar o próprio dinheiro, fazer compras, usar transporte e cuidar da casa, entre outras. Alguns trabalhos mostram melhora nas tendências das AIVD, sem a correspondente melhora nas $\mathrm{AVD}^{4}$, sobretudo entre idosos mais velhos ${ }^{6}$. Resultados de uma meta-análise de estudos conduzidos nos Estados Unidos mostraram que houve uma diminuição na prevalência da incapacidade funcional (AVD e/ou AIVD) ao longo da década de 1990, com uma média anual de declínio entre $-1,55 \%$ e $-0,92 \%{ }^{6}$. Mais recentemente, foi realizado um estudo abrangente para examinar as tendências das AVD nos países da Organização para Cooperação e Desenvolvimento Econômico (OCDE). Entre 12 países, somente cinco mostraram redução na prevalência da incapacidade funcional (Dinamarca, Finlândia, Itália, Holanda e Estados Unidos.). Em três países (Bélgica, Japão e Suécia) houve aumento e em dois países (Austrália e Canadá) a prevalência manteve-se estável ao longo do tempo. Na França e no Reino Unido, resultados de diferentes inquéritos mostraram tendências discrepantes ${ }^{7}$.

O questionário da PNAD inclui somente uma pergunta sobre AVD. Esta se refere ao grau de dificuldade para realizar pelo menos uma entre três atividades da vida diária (alimentar-se, tomar banho e ir ao banheiro), que caracterizam a dependência extrema. Como a pergunta não permite investigar as tendências para cada atividade, as presentes análises foram restritas à informação agrupada. Os resultados destas análises mostraram que não houve mudanças na prevalência da incapacidade funcional entre idosos brasileiros. Essa estabilidade foi observada em todas as regiões do país, assim como em todas as faixas etárias idosas.

No presente trabalho observou-se uma redução significativa na prevalência de artrite ou reumatismo, doença do coração e depressão, e um aumento na prevalência de hipertensão e diabetes. Estes resultados, entretanto, devem ser interpretados com alguma cautela. A pergunta sobre morbidade mudou na PNAD de 2003 e 2008, em relação à de 1998, como mencionado anteriormente. Se a forma como a pergunta foi feita influenciou a resposta, é de se esperar uma mudança abrupta nas estimativas de prevalência entre 1998 e 2003. Isso não ocorreu com relação à hipertensão e ao diabete, cujas prevalências apresentaram aumentos graduais ao longo do tempo, mas para as outras condições as mudanças foram mais marcantes entre 1998 e 2003 do que entre 2003 e 2008. Outro fator que pode ter influenciado os resultados é o uso de serviços de saúde. A probabilidade de saber ter a doença é maior entre aqueles que usam mais serviços de saúde $e^{20-22}$ e as consultas médicas aumentaram ao longo do período do estudo. Entretanto, esse uso tenderia a aumentar as estimativas de prevalência e não o contrário, como observado para quatro das condições investigadas. Com referência à hipertensão e ao diabetes, a associação temporal positiva persistiu mesmo após ajustamentos pelo número de consultas médicas, além da idade e do sexo (não mostrado).

As tendências nos indicadores de usos de serviços de saúde por idosos brasileiros mostram que ocorreram mudanças entre 1998 e 2008. A realização de três ou mais consultas médicas nos 12 meses precedentes aumentou 21\% entre 1998 e 2008. As hospitalizações diminuíram 10\% no mesmo período. $\mathrm{O}$ aumento na realização de três ou mais consultas médicas ocorreu de forma consistente nas três faixas etárias idosas e em todas as regiões brasileiras. A redução das hospitalizações foi observada em todas as regiões do país, assim como na faixa etária idosa mais jovem. Os resultados também mostraram clara predominância de usuários exclusivos do SUS em todo o período estudado, com prevalências variando entre 75\% em 1998 e $72 \%$ em 2008. É interessante observar que as hospitalizações por condições sensíveis à atenção primária pelo SUS (ou seja, internações que podem ser evitadas por ações efetivas da atenção primária) vêm diminuindo na última década. Um estudo recente mostrou que a redução dessas internações estava associada com a cobertura da Estratégia da Saúde da Família. Essa associação - que foi mais forte nas faixas etárias de 60-79 anos do que entre adultos mais jovens - persistiu mesmo após ajustamentos por condições socioeconômicas, indicadores ambientais e indicadores de mortalidade ${ }^{23}$. A redução das hospitalizações observada no presente trabalho é coerente com essas evidências. 


\section{Conclusões}

Os resultados deste trabalho mostram tendências positivas nas condições de saúde da população idosa brasileira em várias dimensões, mas não em todas. As mudanças no uso de serviços de saúde ocorreram como esperado em decorrência da expansão das atividades de atenção primária no Brasil.

\section{Colaboradores}

MF Lima-Costa e James Macinko conceberam o estudo, realizaram a análise dos dados e redigiram o trabalho. DL Matos e VA Camargos prepararam o banco de dados para análise, participaram da análise dos dados e da revisão crítica do manuscrito.

\section{Agradecimentos}

Este trabalho foi desenvolvido como parte das atividades do Núcleo de Estudos em Saúde Pública e Envelhecimento (NESPE) da Fundação Oswaldo Cruz e Universidade Federal de Minas Gerais, enquanto Centro Colaborador em Epidemiologia do Envelhecimento e Saúde do Idoso da Secretaria de Vigilância em Saúde do Ministério da Saúde. Os autores também agradecem as valiosas sugestões do revisor anônimo.

\section{Referências}

1. Palacios R. The future of global ageing. International Journal of Epidemiology 2002; 31(4):786-791.

2. Brasil. Ministério do Planejamento Orçamento e Gestão (MPOG). Instituto Brasileiro de Geografia e Estatística (IBGE). Censos demográficos. Instituto Brasileiro de Geografia e Estatística (IBGE). [site da Internet]. [acessado 2010 ago 7]. Disponível em: http://www. ibge.gov.br

3. Lima-Costa MF, Peixoto SV, Giatti L. Tendências da mortalidade entre idosos brasileiros. Epidemiologia e Serviços de Saúde 2004; 13(4):217-228.

4. Crimmins EM. Trends in the health of the elderly. Annual Review of Public Health 2004; 25:79-98.

5. Brayne C, Mattews FE, McGee MA, Jagger C. Health and ill-health in the older population in England and Wales: the Medical Research Council Cognitive Function and Ageing Study (MRC CFAS). Age and Ageing 2001; 30(1):53-62.

6. Freedman VA, Martin LG, Schoeni RF. Recent trends in disability and functioning among older adults in the United States. JAMA 2002; 288(24):3137-3146

7. Gaétan L, Balestat G. The Disability Study Expert Group Members. Trends in severe disability among elderly people: assessing the evidence in 12 OECD countries and the future implications. Diretorate for employment, labour and social affairs. Paris: OECD Health Working Papers; 2007.

8. Gu D, Dupre ME, Warner DF, Zeng Y. Changing health status and health expectancies among older adults in China: gender differences from 1992 to 2002. Soc Sci Med 2009; 68(12):2170-2179.

9. Jang SN, Kim DH. Trends in the health status of older Koreans. J Am Geriatr Soc 2010; 58(3):592-598.

10. Lima-Costa MF, Loyola Filho AI, Matos DL. Tendências nas condições de saúde e uso de serviços de saúde entre idosos brasileiros: um estudo baseado na Pesquisa Nacional por Amostra de Domicílio (1998, 2003). Cad Saude Publica 2007; 23(10):24672478. 
11. Lima-Costa MF, Barreto SM, Giatti L. Condições de saúde, capacidade funcional, uso de serviços de saúde e gastos com medicamentos da população idosa brasileira: um estudo descritivo baseado na Pesquisa Nacional por Amostra de Domicílio (PNAD). Cad Saude Publica 2003; 19(3):735-743.

12. Instituto Brasileiro de Geografia e Estatística (IBGE). Pesquisa Nacional por Amostra de Domicílios. Acesso e utilização de serviços de saúde: 1998: Brasil/ IBGE, Departamento de Emprego e Rendimento. Rio de Janeiro: IBGE; 2000.

13. Instituto Brasileiro de Geografia e Estatística (IBGE). Pesquisa Nacional por Amostra de Domicílios. Síntese de indicadores 2003. Coordenação de Emprego e Rendimento. Rio de Janeiro: IBGE; 2004.

14. Instituto Brasileiro de Geografia e Estatística (IBGE). Pesquisa Nacional por Amostra de Domicílios. Um panorama da saúde no Brasil. Acesso e utilização de serviços, condições de saúde e fatores de risco e proteção à saúde 2008: Brasil/IBGE, Coordenação de Trabalho e Rendimento. Rio de Janeiro: IBGE; 2010.

15. Silva PLN, Pessoa DGC, Lila MF. Análise estatística de dados da PNAD: incorporando a estrutura do plano amostra. Cien Saude Colet 2002; 7(4):659-670.

16. Lima-Costa MF, Firmo JOA, Uchoa E. A estrutura da auto-avaliação da saúde entre idosos: Projeto Bambuí. Rev Saude Publica 2004; 38(6):827-834.

17. Jylhä M. What is self-rated health and why does it predict mortality? Towards a unified conceptual model. Soc Sci Med 2009; 69(3):307-316.

18. Hennessy CH, Moriarty DG, Zack MM Scherr PA, Brackbill R. Measuring health-related quality of life for public health surveillance. Public Health Rep 1994; 109(5):655-672.
19. Health Interview Surveys: Towards International Harmonization of Methods and Instruments (WHO Regional Publications, European Series, No. 58). Copenhagen, Denmark: World Health Organization Regional Office for Europe; 1996.

20. Martin LM, Leff M, Calonge N, Garrett C, Nelson DE. Validation of self-reported chronic conditions and health services in a managed care population. Am J Prev Med 2000; 18(3):215-218.

21. Bowlin SJ, Morrill BD, Nafziger AN, Lewis C, Pearson TA. Reliability and changes in validity of selfreported cardiovascular disease risk factors using dual response: The Behavioral Risk Factor Survey. Journal of Clinical Epidemiology 1996;49(5):511-517.

22. Lima-Costa MF, Peixoto SV, Firmo JOA. Validade da hipertensão arterial auto-referida e seus determinantes (Projeto Bambuí). Rev Saude Publica 2004. 38(5):637-642.

23. Macinko J, Oliveira VB, Turci MA, Guanais FC, Bonolo P, Lima-Costa MF. The Influence of Hospital and Primary Care Supply on Ambulatory Care Sensitive Hospitalizations Among Adults in Brazil, 1999-2007. American Journal of Public Health 2010; (Ahead of print: 10.2105/AJPH.2010.198887).

Artigo apresentado em 02/09/2010

Aprovado em 05/05/2011

Versão final apresentada em 10/05/2011 